\title{
Long-Term Electricity Contract Optimization with Demand
}

\section{Uncertainties}

\author{
Pang Chan ${ }^{{ }^{2} \text {, }}$ Chi-Wai Hui ${ }^{\text {a }}$, Wenkai Li ${ }^{\text {a }}$ \\ Haruo Sakamoto ${ }^{b}$, Kentaro Hirata ${ }^{b}$ \\ $\mathrm{Pu} \mathrm{Li}^{\mathrm{c}}$
}

${ }^{a}$ Department of Chemical Engineering, Hong Kong University of Science and Technology, Clear Water Bay, Hong Kong

\begin{abstract}
${ }^{\mathrm{b}}$ Process Development and Design Lab., Process Systems Engineering and Production Technologies, Research \& Technology Development Division, Mitsubishi Chemical Group Science \& Technology Research Center, Inc. 1, Toho-cho,Yokkaichi, Mie 510-8530, Japan

${ }^{\mathrm{c}}$ Institut für Prozess- und Anlagentechnik, Technische Universität Berlin, KWT 9 10623 Berlin, Germany
\end{abstract}

\begin{abstract}
This paper presents a study on selecting electricity contracts for a large-scale chemical production plant, which requires electricity importation, under demand uncertainty. Two common types of electricity contracts are considered, Time Zone (TZ) contract and Loading Curve (LC) contract. A multi-period linear probabilistic programming model is adopted for the contract selection and optimization. Hence, by using the probabilistic programming, a solution procedure is proposed that allow users to determine the best electricity contract according to their desired confident level of the uncertainties. In addition, due to the fact that the demand of product is uncertain, if one considers the overage and shortage of the products in the market as well, an interesting result can be obtained. The methodology is explained in the paper.
\end{abstract}

\footnotetext{
*Corresponding author 7004254@cc.m-kagaku.co.jp
} 
Keywords: Electricity Contract, Optimization, Uncertainty, Probabilistic Programming, Confidence Level.

\section{Introduction}

Deterministic models are commonly used for production planning in which it is assumed that the prices, demand of products and the availabilities of raw materials, etc., are known. In fact, unlike process operations, market conditions in the future time horizon, in which process operation is planned, present uncertainties that play an important role in a planning process. Many studies on this topic have been conducted in the recent years [1].

Sources of uncertainty in production planning can be categorized as short-term or long-term [2] based on their time frames. Short-term uncertainty may include day-today processing variations, cancelled/rushed orders and equipment failure etc. Longterm uncertainty refers to raw material/final product unit price fluctuations, demand variations and production rate changes over longer time frames.

\section{Nomenclature}

\section{Sets}

i, $(i=1,2, \ldots . .12) \quad$ Month

$\mathrm{j},(\mathrm{j}=1,2,3) \quad$ Shift (1: Day, 2 : Night and 3: Mid-night)

\section{Variables}

$y_{\mathrm{ij}}$

$q_{\mathrm{ij}}$

$X_{\mathrm{i}}$

$\mathrm{x}_{\mathrm{i}}$

$\mathrm{P}_{\mathrm{i}}$

$\mathrm{S}_{\mathrm{D}}$

$\mathrm{S}_{\mathrm{N}}$
Amount of $\mathrm{P}$ produced at different $\mathrm{i}$ and $\mathrm{j}(\mathrm{t} / \mathrm{shift})$

Amount of EL imported at different $i$ and $j$ (MW)

Demand, a random variable at month i (t)

Realized demand at month i ( $\mathrm{t}$ )

$\sum_{j} y_{i j} \times S L_{j}$, Production target at month i (t)

Maximum electricity importation during the whole contract period at D shift (MW)

Maximum electricity importation during the whole contract period at $\mathrm{N}$ shift (MW) 
$\mathrm{S}_{\mathrm{M}}$

TEL

ELFix

\section{Parameters}

$S L_{\mathrm{j}}$

$\mathrm{r}$

$\mathrm{C}_{\mathrm{ij}}$

$\mathrm{q}_{\mathrm{ijMin}}$

$\mathrm{q}_{\mathrm{ijMax}}$

$a_{D}$

$a_{N}$

$a_{M}$

$\mathrm{a}_{\mathrm{T}}$

$\alpha$

$\mathrm{MA}_{\mathrm{j}}$

ELMAX $_{j}$

$\theta$

$\sigma$

$\phi$

$\mathrm{g}\left(\mathrm{x}_{\mathrm{i}}\right)$

$\Phi$

$\Phi^{-1}$

Z
Maximum electricity importation during the whole contract period at M shift (MW)

Annual electricity importation, $\sum_{i} \sum_{j} q_{i j} \times S L_{j}(\mathrm{MWH})$

Electricity fixed cost (MYEN)

Length of shift $\mathrm{j}(8 \mathrm{~h} / \mathrm{shift})$

Product price $\$ 0.035$ (MYEN/t)

Cost parameter of EL at different $i$ and $j$ (MYEN/MW)

Lower bound of electricity importation in each shift (MW)

Upper bound of electricity importation in each shift (MW)

Parameter corresponding to the max electricity importation at

D shift. Value used in the case study: 14 (MYEN/MW)

Parameter corresponding to the max electricity importation at

$\mathrm{N}$ shift. Value used in the case study: 3 (MYEN/MW)

Parameter corresponding to the max electricity importation at

M shift. Value used in the case study: 1.5 (MYEN/MW)

Parameter corresponding to the total electricity importation.

Value used in the case study: 0.0025 (MYEN/MWH)

Confidence Level

Max. capacity of production at shift $\mathrm{j}$ (30 $\mathrm{t}$ per shift)

Maximum electricity importation limit at $S_{D}, S_{N}$ and $S_{M}$ (MW)

Mean of demand at month i ( $\mathrm{t})$

Standard deviation of demand at month i $(\mathrm{t})$

Probability density function

Probability density function (pdf) of $X_{i}, g\left(x_{i}\right)=d \Phi\left(x_{i}\right) / \mathrm{dx}_{\mathrm{i}}$

Cumulative distribution function (cdf) of X

Inverse of cumulative distribution function

$(\mathrm{P}-\theta) / \sigma$

So far, very few applications of optimization under uncertainty have been made in chemical process operations [3], especially for the electricity contract optimization for industrial process operations. In fact, selecting a right electricity contract for a largescale chemical production plant, which requires electricity importation, is very 
important for minimizing overall energy cost. The amounts of steam and electricity usages in a chemical production site are fluctuated due to the uncertain market conditions. These fluctuations on one the hand can be adsorbed by the utility system, or on the other hand balanced by the importation of electricity.

Importing electricity from an external supplier requires a long-term contract that normally covers a period of one to three years. Several types of electricity supply contracts are designed by the suppliers that target for different type of customers. The contract fee often depends on the overall of annual consumption, the maximum consumption rates at the peak, medium and low demand periods and the emergency backup, etc. In the existence of uncertain factors such as product demands, the determination of a long-term electricity supply contract and its values are definitely affected. This paper is going to present a mathematical model and a systematic procedure for selecting an optimal electricity long-term contract under uncertainty. In this model, the demand of product is assumed to be uncertain. By considering the overage and shortage of the products in the market, an interesting result is found for decision-making.

Notice that the overage products refer to the products that cannot be absorbed by the market and disposed eventually; while the shortage products are not compensated from the outside environment in order to fulfil the market demand. No inventory is provided for holding such kind of unsold products.

\section{Probabilistic approach}

There have been two general stochastic approaches [4] to solve uncertain problems: one is the two-stage stochastic programming and the other is chance constrained programming (also known as probabilistic approach). In authors [5] argued that the choice of the appropriate method is content-dependent, with no single theory being sufficient to model all kinds of uncertainty. It is noticed that with both solution strategies there have been, until now, no suitable approaches to the nonlinear problems. In this paper, the probabilistic approach is mainly concentrated and elaborated in the next section.

From the application point of view, the method of chance constrained programming to linear, nonlinear and dynamic problems has been developed and applied to many disciplines like finance and management $[6,7]$ in recent years. However few 
applications have been made in chemical process operations [3]. Usually, the probabilistic approach seeks to satisfy the constraints involved by a pre-determined confidence level, denoted as $\alpha(0 \leq \alpha \leq 1)$, based on the known probability density or cumulative distribution of the uncertain variables $[4,6]$. Therefore, this approach can provide essential information on the economical achievements as a function of the desired confidence level. For complex plant operations under multiple uncertainties, the sources of risk that have the most significant impacts on the profitability can be identified $[8,9]$.

Using the probabilistic approach, a stochastic programming problem under chance constraints is formulated for optimization. In many cases, it can be relaxed to an equivalent deterministic LP or NLP problem. The essential challenge lies in the computation of the probabilities of holding the constraints. Its unique feature is that the resulting solution ensures the probability of satisfying constraints. The solution leads to an expected optimal value of the objective function by searching for the decision in a feasible region at a given confidence level. In addition, the term -Confidence Level can be defined into two ways: the first one is related to the constraint that the amount of the product being produced can satisfy the market demand. For example, we have $90 \%$ confidence to fulfil the market demand for the produced product. The second definition is related to the constraint that the amount of the generated product can be sold to the market. For example, we have confidence to sell out $90 \%$ of product. Different people have different interpretation to this term; users should make clear before using it. In this work, we adopt the former definition throughout the paper. Since $\alpha$ has to be defined by the decision-marker, it is possible to select different confidence levels and make a compromise between the objective value and the risk of constraint violation.

\section{A Case study}

\subsection{Problem Definitions}

A motivating example shown in Figure 1 is used for illustrating the problem and the proposed solution method. In this problem, process " $\mathrm{A}$ " is going to produce product by mainly consuming electricity. Each ton of product being produced consumes 850 
$\mathrm{kW}$ of electricity. The price of raw material is negligible in this example. The product demand from the market is under uncertain.

\subsection{Uncertain market demand}

In this case, the objective is to maximize the profit. However, due to the uncertain market demand, i.e. the demand of product is an uncertain variable, $\mathrm{X}$, cannot be determined a priori. This results in an overage of product if the production rate is overestimated or a shortage of product if the production rate is underestimated. Both situations will lead to a profit reduction or even a negative profit for the production plant. Fortunately, this unhealthy condition can be remedied by taking the demand uncertainty into consideration. Let assume the uncertainties of product demands having a normal distribution. As shown in Table 1, the distribution is described with four seasons: Winter, Spring, Autumn and Summer, each season has its demand distribution, while the demand distribution in autumn and spring are the same. Figure 2 represents the density function of the uncertain variable in different seasons.

\subsection{Electricity contract and cost calculation}

As the production of product requires electricity, based on the demand distribution, the overall electricity imported from a power plant can be estimated. Actually, this is an important step to have a general idea regarding the amount of electricity being consumed before signing a contract with the power plant. In general, there are two common types of electricity contracts provided by the power plant [10], which are Time Zone (TZ) contract and Loading Curve (LC) contract, users can base on contractual information provided by the electricity suppliers or their estimated electricity purchase amount to choose an optimal contract in order to minimize the electricity cost. The characterization of the two kinds of contracts is as follows:

TZ Contract: Electricity supply is classified into several types (e.g. peak, normal and off peak) according to the demand in different time shifts and periods. Simple regulations or restrictions are employed in the contract to encourage the users to consume more electricity during the off peak periods, such as different prices, intake maximum and/or minimum values at different time periods. 
LC Contract: Besides different electricity types, the LC contract has more restrictions on the purchase of customers, so that the electricity company can plan its operation easier. The customers are requested to follow the upper and lower importation limits at all time shifts, the annual overall purchasing amount, and some other constraints for the contracted year. If any of these restrictions is violated, penalties will be charged on the customers. The customers however are rewarded by a lower electricity tariff and are guaranteed by the contracted demand.

For the calculation of the electricity contract fee, the cost is divided into two categories, variables cost and fixed cost. Different kinds of variable cost calculation method are adopted in current commercial practice, and they can be briefly classified into three types. First, the variable cost only has one fixed value. The variable cost only depends on the intake amount and the fixed cost parameter. Second, the electricity company defines a cost profile, which divides the electricity purchased amount into different ranges, and each range has its own cost parameter. The variable cost will then be calculated by the purchased amount multiplying with its corresponding cost parameter. Third, the cost parameters vary according to the time shifts (D: Day, N: Night \& M: Mid-night). Thus, the variable cost is calculated by the total importing amount and the time period of the importation. In this paper, both contracts are using the third type to calculate the variable cost.

Besides the variable cost, fixed cost also takes a very important position in the electricity supply contract. Fixed cost is a fixed annual fee that the plant site has to pay every year, which depends on the maximum importation peak on the contracted period. Allowing a higher maximum import level, the production site might have a higher flexibility for production and/or maintain its production even during utility equipment failure. On the other hand, a higher maximum importation rate increases the fixed cost. The profit gain due to higher productivity may not be able to compensate the additional payment of the electricity contract. Thus, careful consideration of the electricity contract is necessary.

For the $\mathrm{TZ}$ contract, the contract fee contains the variable cost only; the calculation method is already mentioned above by using the third method. The upper bound of $25 \mathrm{MW}$ importation rate in each shift is applied for the whole contracted period. Notice that there is no lower bound of annual importation for the TZ contract. 
For the LC contract, the upper bound of importation rate is different in different seasons and time shifts. The contract fee contains two parts, variable cost and fixed cost. The fixed cost calculation follows the Eq. (1) stated below.

Those parameters and conditions of the two contracts offered by the power plant are shown in the Table 2, 3 and 4.

ELFix $=a_{D} S_{D}+a_{N} S_{N}+a_{M} S_{M}+a_{T} T E L$

In brief summary, the equations to calculate the contract fee of $\mathrm{TZ}$ and $\mathrm{LC}$ contracts are shown as follows:

ELcost $\mathrm{TZ}=\sum_{i j}\left[(\text { Amount of EL imported })_{j} \times(\cos t \text { parameter })_{j}\right]_{i}$

ELcost $_{\mathrm{LC}}=\sum_{i j}\left[(\text { Amount of EL imported })_{j} \times(\cos t \text { parameter })_{j}\right]_{i}+$ ELFix

Let us use two simple examples to show the calculation of the electricity cost based on the different contract types (TZ or LC), and see which contract is optimal. The electricity prices are provided in Table 2.

Example 1: The annual electricity importation and the upper bound at each shift are shown Table 5. Then the electricity cost can be calculated as follows:

$$
\begin{aligned}
\text { ELcost }_{\mathrm{TZ}}= & 500 * 0.022+1000 * 0.011+2000 * 0.0077 \\
= & 37.4 \mathrm{MYEN} \\
\text { ELcost }_{\mathrm{LC}}= & (500 * 0.018+1000 * 0.006+2000 * 0.003) \\
& \quad+(0.3 * 14+0.4 * 3+0.7 * 1.5+3500 * 0.0025) \\
= & 36.2 \mathrm{MYEN}
\end{aligned}
$$

It means selecting the $\mathrm{LC}$ contract is better than $\mathrm{TZ}$ contract. 
Example 2: The annual electricity importation and the upper bound at each shift are shown Table 6. Then the electricity cost with different contracts can be calculated as follows:

$$
\begin{aligned}
\text { ELcost }_{\mathrm{TZ}} & =300 * 0.022+700 * 0.011+1500 * 0.0077 \\
& =25.85 \mathrm{MYEN}
\end{aligned}
$$

$$
\begin{aligned}
\text { ELcost }_{\mathrm{LC}}= & (300 * 0.018+700 * 0.006+1500 * 0.003) \\
& +(0.3 * 14+0.4 * 3+0.7 * 1.5+2600 * 0.0025) \\
= & 27.05 \mathrm{MYEN}
\end{aligned}
$$

In this case $\mathrm{TZ}$ contract would be better!

These two examples demonstrate the choice of an electricity contract depends on the annual electricity importation. The customers should have a good estimation of electricity amount to be consumed during the contracted period before making a decision.

However, it might turn out that the contract being chosen may not be the best due to the uncertain factors. An uncertain demand, one of the uncertain factors, definitely affects the rate of electricity importation during the contracted period. Hence, the customers might suffer from the high electricity costs if the demand uncertainty is neglected.

\section{Production planning under uncertainty}

Assuming that this is a 12 -month, $i=1, \ldots, 12$, planning period production with 3 operating shifts, $j=\mathrm{D}, \mathrm{N}, \mathrm{M}$ in a day ( 8 hours each shift). The objective of this study is to generate a production plan for maximum profit with considerations of uncertain market demand and electricity prices together with the electricity contract information.

Table 7 shows the amount of product that satisfies the market demand at the predefined confidence level. Take March at $\alpha=0.3$ as an example to demonstrate the calculation of the data in Table 7. Using the mean and standard deviation of March distribution, a cumulative density function curve can be obtained and shown in 
Figure 3. At $\alpha=0.3$, the product amount that satisfies the market demand is determined, which is $9905.61 \mathrm{t}$. This method can be applied for finding other production targets at different confidence levels.

\subsection{Mathematical Formulation}

The optimization problem is formulated with two different stochastic constrained models. The first model is used for Electricity Cost calculation and the second is for Revenue calculation. The electricity cost calculation is based on the defined confidence level; optimization is required to find out the optimal imported electricity amount in each shift. After that the revenue calculation can then be easily calculated based on the optimal imported electricity amount. There is no optimization required for revenue calculation. Let us first define the objective function in a mathematical way:

Obj. function Max $\quad$ Profit $=($ Revenue - Electricity cost $)$

The solutions for calculating the Revenue and Electricity cost are explained in detail in the following two sections. The electricity cost calculation is discussed first.

\subsubsection{The electricity cost}

To model the calculation of the electricity cost, let $q_{i j}$ and $y_{i j}$ denote the positive variables of electricity input and product output in different months (i) and shifts $(j)$, respectively. $C_{i j}$ and $S L_{j}$ represent the electricity price and shift length. ELFix shown in Eq. (1) is comprised of some terms, let $S_{D}, S_{N}$ and $S_{M}$ denote the maximum electricity demand at $\mathrm{D}, \mathrm{N}$ and $\mathrm{M}$ shifts corresponding to their parameters $a_{D}, a_{N}$ and $a_{M} . S_{D}, S_{N}$ and $S_{M}$ are positive variables to be determined by the model. TEL stands for the total electricity importation during the contracted period corresponding to the parameter, $a_{T}$. The model is implemented in the framework of GAMS [11] and solved it with the solver OSL [12]. In summary, the electricity cost corresponding to a predefined confidence level can be obtained by solving the following model: 
Min Electricity cost $=\sum_{i} \sum_{j}\left(\mathrm{C}_{\mathrm{ij}} \mathrm{q}_{\mathrm{ij}}\right) \times S L_{j}+$ ELFix

s.t

$$
\begin{aligned}
& q_{i j}=0.85 y_{i j} \\
& q_{i j M i n} \leq q_{i j} \leq q_{i j M a x} \\
& \operatorname{Pr}\left(\sum_{j}\left(y_{i j} \times S L_{j}\right) \geq X_{i}\right) \geq \alpha \\
& y_{i j} \leq M A_{j} \\
& T E L \geq 100,000 \\
& S_{D}, S_{N}, S_{M} \leq E L M A X_{j} \\
& q_{i j}, y_{i j} \geq 0 \\
& i=1, \ldots, 12 \text { and } j=1, \ldots, 3
\end{aligned}
$$

Eq. (2.1) minimizes the variable and fixed cost of electricity. Eq. (2.2) enforces the material balance, it means that $850 \mathrm{~kW}$ of electricity required producing one ton of product per hour; while Eq. (2.3) ensures that the supply of electricity at each shift would not under/exceed the assigned lower/upper bound $\left(\mathrm{q}_{\mathrm{ijMin}} / \mathrm{q}_{\mathrm{ijMax}}\right)$ value by the contract (note that different contracts have different $\mathrm{q}_{\mathrm{ijMin}}$ and $\mathrm{q}_{\mathrm{ijMax}}$ values). Eq. (2.4) determines the production rate of $\sum_{j}\left(y_{i j} \times S L_{j}\right)$ at each month, which fulfils the market demand by the defined confidence level (i.e. the probability of production rate greater than the demand should be equal or greater than the defined confidence level). Eq. (2.5) ensures that the production rate in each shift should not exceed the maximum plant capacity, $\mathrm{MA}_{\mathrm{j}}$. Eq. (2.6) restricts the plant to import at least 100,000MWH during the whole contract period. This constraint is applied for the LC contract only (note that $T E L=\sum_{i} \sum_{j} q_{i j} \times S L_{j}$ ). Eq. (2.7) restricts the electricity purchase below the upper limit for electricity importation at each shift, ELMAX $\mathrm{E}_{\mathrm{j}}$ The problem defined above is called stochastic probabilistic (or constrained) programming problem. To solve this problem with an available optimization routine, the probabilistic constraint must be relaxed by replacing Eq. (2.4) with the following (Please see the appendix part I): 


$$
\sum_{j}\left(y_{i j} \times S L_{j}\right) \geq \Phi^{-1}(\alpha)
$$

Hence, the only difference between the stochastic and relaxed programming is Eq. (2.4) and (2.4a), in which the stochastic constraint is relaxed to an equivalent deterministic form. The $\Phi^{-1}$ means the inverse of cumulative density function with $\Phi(\infty)=1$ [13]. The meaning of $\Phi$ and $\Phi^{-1}$ would be explained in more detail in the following section.

\subsubsection{The Revenue}

The revenue corresponding to the same confidence level is calculated as follows:

$$
\begin{gathered}
\text { Revenue }=r \times \sum_{i} \int_{0}^{\infty} \min \left(P_{i}, x_{i}\right) g\left(x_{i}\right) d x_{i} \\
\text { s.t } P_{i}=\sum_{j}\left(y_{i j} \times S L_{j}\right)
\end{gathered}
$$

$$
i=1, \ldots, 12 \text { and } j=1, \ldots, 3
$$

$\mathrm{r}$ refers to the product price. $\mathrm{P}_{\mathrm{i}}$ equals to the monthly production target. The demand, $x$, is assumed to have a continuous normal distribution that is described by two parameters, the mean $\theta$ and the standard deviation $\sigma$. Here $g(x)$ represents the probability density function (pdf). The cumulative distribution function (cdf) is the integral of the density function, i.e. $\int_{0}^{\infty} g(x) d x$. Hence, the expected demand $(\theta)$ can be calculated by multiplying the demand, $x$, in the integral. Hence, the term $\int_{0}^{\infty} x g(x) d x$ represents the expected demand in each month. However, by replacing it with $\int_{0}^{\infty} \min (P, x) g(x) d x$, it becomes the expected amount of product sold to the market, i.e. the smaller value among the demand and production rates would be selected for the integration. In the other words, products in overage or shortage are considered in the revenue calculation. In fact, this integral is based on the assumptions that the overage products that are not sold to the market would not be stored and 
disposed eventually, while the shortage products would not be imported from outside companies to fulfil the market demand and no inventory holding as well. For other cases that do not apply for the above assumptions, the expected result is not valid.

Let take one simple example to illustrate the meaning of $\int_{0}^{\infty} \min (P, x) g(x) d x$. Assume $x=13000 \mathrm{t}$ and $P=12000 \mathrm{t}$. Since the demand is $13000 \mathrm{t}$, and the plant can only produce $12000 \mathrm{t}$, the actual amount of the product sold to the market is $12000 \mathrm{t}$ only. Therefore, by multiplying the corresponding price, $r$, in the integral, the expected revenue can be calculated by using Eq. (3.1).

In addition, Eq. (3.1) is also related with Eq. (3.2), in which $y_{i j}$ is already determined from the electricity cost calculation. This constraint is used to define the production target, $P$, in order to fulfil the market demand by the defined confidence level. Hence, by applying the basic probability techniques [14], Eq. (3.1) can be solved by available commercial routines, and the problem can be relaxed to the following deterministic form (Please see the appendix part II and III):

$$
\begin{gathered}
\text { Revenue }=r \times \sum_{i}\left\{\theta_{i} \Phi\left(z_{i}\right)+P_{i}\left(1-\Phi\left(z_{i}\right)\right)-\sigma_{i} \phi\left(z_{i}\right)\right\} \\
\text { s.t } P_{i}=\sum_{j}\left(y_{i j} \times S L_{j}\right)
\end{gathered}
$$

where $\mathrm{z}=(\mathrm{P}-\theta) / \sigma$$$
\mathrm{i}=1, \ldots, 12 \text { and } \mathrm{j}=1, \ldots, 3
$$

where $\Phi, \phi$ represent the cdf and pdf of the standard normal distribution, respectively. It is noted that Eq. (4.1) is obviously easier to solve than Eq. (3.1) since it does not have an integral term. The value of $\Phi(z), \phi(z)$ can be easily obtained by calling standard routines. Hence, at different confidence levels, the expected revenue can be calculated. It is remind that the derivation of equations (2.4) and (3.1) can be found in the Appendix and the meaning of each symbol shown in these two models can be found in the Nomenclature.

Although individual models are set up for calculating the electricity cost and revenue, they can be combined as one model for optimization indeed. The revenue model, 
however, doesn't involve the optimization skill; therefore it is possible to consider both models separately in order to make a clearer picture.

\subsection{Results and Discussions}

Table 8 and 9 show the results of revenue and electricity cost models.

Here we pick up $\alpha=0.9$ as an example to illustrate the calculation of the expected revenue and electricity cost for $\mathrm{TZ}$ and $\mathrm{LC}$ contracts.

The Revenue: Using constrained equation (2.4a), by fixing the confidence level at 0.9 , the production rate can be determined and shown in Table 10.

Then, by using the Eq. (4.1), the expected revenue can be calculated as follows:

$$
\begin{aligned}
& \text { Re venue }=0.035 \times 4 \times \\
& \quad\left[12000 \Phi\left(\frac{12281.94-12000}{220}\right)+12281.94\left(1-\Phi\left(\frac{12281.94-12000}{220}\right)\right)-220 \phi\left(\frac{12281.94-12000}{220}\right)\right. \\
& \quad+10000 \Phi\left(\frac{10230.68-10000}{180}\right)+10230.68\left(1-\Phi\left(\frac{10230.68-100000}{180}\right)\right)-180 \phi\left(\frac{10230.68-10000}{180}\right) \\
& \left.\quad+13000 \Phi\left(\frac{13320.39-13000}{250}\right)+13320.39\left(1-\Phi\left(\frac{13320.39-13000}{50}\right)\right)-250 \phi\left(\frac{13320.39-13000}{250}\right)\right] \\
& =4895.69 \text { MYEN }
\end{aligned}
$$

The electricity cost: At $\alpha=0.9$, the optimal annual electricity importation at each shift and the maximum electricity importation after optimization are shown in the Table 11.

Then the cost can be computed as follows:

$$
\begin{aligned}
\operatorname{ELcost}_{\mathrm{TZ}}= & 292 * 0.021+48540.74 * 0.011+73000 * 0.0077 \\
= & 1102.17 \mathrm{MYEN} \\
\text { ELcost }_{\mathrm{LC}}= & (9775.92 * 0.018+39056.31 * 0.006+73000 * 0.003) \\
& +(7.18 * 14+15 * 3+25 * 1.5+121832.23 * 0.0025
\end{aligned}
$$


$=1116.85 \mathrm{MYEN}$

Hence, Profit under TZ contract $=4895.69-1102.17=3793.52 \mathrm{MYEN}$

Profit under LC contract $=4895.69-1116.85=3778.84$ MYEN

The figures 4 and 5 indicate the electricity importation under the TZ and LC contracts at $\alpha=0.9$. Both of them show the company should import less electricity at D and $\mathrm{N}$ shifts and the amount pointed to the upper bound at $\mathrm{M}$ shift. The reason is due to the high electricity price corresponding to $\mathrm{D}$ and $\mathrm{N}$ shifts, the company should avoid consuming electricity at these two shifts in order to minimize the electricity cost. Actually, this is a typical profile for the LC/TZ contract in the industrial practice. A similar result could be obtained at various confidence levels for both contracts.

Table 12 and figure 6 show the results of the two profits by applying the TZ and LC contracts. It can be seen that both contracts have the optimal points at one particular confidence level. It is interesting to point out that this result cannot be found from some conventional uncertainty studies. In conventional studies, if $\alpha$ is toward zero, the demand inequality of Eq. (2.4a) is meaningless, which means that the optimization can be made without this constraint. Then the profit remains maximal at $\alpha$ equals to 0 . In other words, the Profit against confidence level curve is always descending.

However, this is only correct when we use the expectation of demand for calculating the revenue. If we use Eq. (4.1) to calculate the revenue instead of using expectation, the result shows a maximum point at one particular confidence level. Therefore, when we only adopt the TZ contract for the production planning, we should choose $\alpha$ $=0.7$ for the production target in order to maximize the profit. The same situation is applied for the LC contract, but the optimal confidence level is located at 0.4. It is noted that there is a considerable drop of profit at $\alpha$ from 0.9 to 0.9995 . The reason is that, if the confidence level is increased from this range, more products may turn into the overage products and cannot be sold to the market eventually. Thus the profit decreases. This is the same logic for explaining the considerable change of profit at the lower confidence level.

However, it is interesting to note that if both contracts are taking into consideration simultaneously, there is an intersecting point. The two curves intersect at the $\alpha=0.76$. Hence, if a desired confidence level chosen by the decision-marker is smaller than 
0.76 , the profit obtained under the LC contract is higher than the TZ contract and vice versa. It turns out that if a company has planned its production schedule with the confidence level lower than 0.76 , there would be less than $76 \%$ probability for the products to satisfy the market demand, and then it is better to choose the LC contract; otherwise the $\mathrm{TZ}$ contract would be the wise choice for the confidence level above 0.76 .

The selection of the right electricity contract is sensitive to the prices of electricity. Considering another set of electricity price for the TZ contract with D: 20000 YEN/MWH, N: $10500 \mathrm{YEN/MWH}$ and M: $7500 \mathrm{YEN/MWH,} \mathrm{in} \mathrm{which} \mathrm{the} \mathrm{price} \mathrm{at}$ all shifts are decreased a little bit compared with those prices given in Table 2. The profit changes with confidence level are shown in figure 7 . It is found that by decreasing the electricity price for the $\mathrm{TZ}$ contract, the profit definitely increases. However this shifts the intersecting point from the $\alpha=0.76$ to $\alpha=0.26$. This means that if the company has planned its production schedule with the confidence level lower than 0.26 , then it is better to choose the LC contract; otherwise the TZ contract would be a better choice. The solution can provide different information for the decision-marker to analysis and choose the electricity contract based on the defined confidence level at different combination of contract values.

\section{Conclusions}

In this paper, the probabilistic programming model is used for selecting a right electricity importation contract under demand uncertainties. The selection of electricity contract and the optimization of contracted terms should be carefully studied for every individual consumer under his/her desired confidence level. Instead of letting the optimizer determine the selection of electricity contract, this paper presented an approach that allows the users to make decisions based upon the desired confidence level of satisfying the uncertain demands. A case study is used to illustrate the scope of the approach.

\section{Acknowledgement}

The authors would like to acknowledge financial support from the Research Grant Council of Hong Kong (DAG00/01.EG05), the National Science Foundation of China 
(Grant No. 79931000) and the Major State Basic Research Development Program (G2000026308). 


\section{Appendix}

\section{Part I.}

From Eq. (2.4), the production rate greater than the demand should be equal or greater than the predefined confidence level. To describe the random variables, $X_{i}$, it is assumed that the pdf of each variable is known.

$$
\begin{aligned}
& \operatorname{Pr}\left(\sum_{j}\left(y_{i j} \times S L_{j}\right) \geq X_{i}\right) \geq \alpha \\
& \operatorname{Pr}\left(X_{i} \leq \sum_{j}\left(y_{i j} \times S L_{j}\right)\right) \geq \alpha \\
& \Phi\left(\sum_{j}\left(y_{i j} \times S L_{j}\right)\right) \geq \alpha \\
& \sum_{j}\left(y_{i j} \times S L_{j}\right) \geq \Phi^{-1}(\alpha)
\end{aligned}
$$$$
\mathrm{i}=1 \ldots 12
$$

\section{Part II.}

In Eq. (3.1), the value of the expected revenue with production target $P_{i}$ needs to be evaluated, this is derived in the follows:

$$
\begin{aligned}
& r \times \sum_{i} \int_{0}^{\infty} \min \left(P_{i}, x_{i}\right) g\left(x_{i}\right) d x_{i} \\
& =r \times \sum_{i}\left(\int_{0}^{P_{i}} x_{i} g\left(x_{i}\right) d x_{i}+\int_{P_{i}}^{\infty} P_{i} g\left(x_{i}\right) d x_{i}\right) \\
& =r \times \sum_{i}\left(\int_{0}^{\infty} x_{i} g\left(x_{i}\right) d x-\int_{P_{i}}^{\infty} x_{i} g\left(x_{i}\right) d x_{i}+\int_{P_{i}}^{\infty} P_{i} g\left(x_{i}\right) d x_{i}\right) \\
& =r \times \sum_{i}\left(\theta_{i}-\int_{P_{i}}^{\infty} x_{i} g\left(x_{i}\right) d x_{i}+\int_{P_{i}}^{\infty} P_{i} g\left(x_{i}\right) d x_{i}\right) \\
& =r \times \sum_{i}\left(\theta_{i}-\int_{P_{i}}^{\infty}\left(x_{i}-P_{i}\right) g\left(x_{i}\right) d x_{i}\right) \\
& =r \times \sum_{i}\left(\theta_{i}-\left[\left(\theta_{i}-P_{i}\right)\left(1-\Phi\left(z_{i}\right)\right)+\sigma_{i} \phi\left(z_{i}\right)\right]\right) \\
& =r \times \sum_{i}\left(\theta_{i} \Phi\left(z_{i}\right)+P_{i}\left(1-\Phi\left(z_{i}\right)\right)-\sigma_{i} \phi\left(z_{i}\right)\right)
\end{aligned}
$$




\section{Part III.}

From Eq. (A.4) to Eq. (A.5) can be derived by considering the following term:

$$
\begin{aligned}
& \sum_{i}\left(\int_{P_{i}}^{\infty}\left(x_{i}-P_{i}\right) g\left(x_{i}\right) d x_{i}\right) \\
& =\sum_{i}\left(\int_{P_{i}}^{\infty}\left(x_{i}-\theta_{i}\right) g\left(x_{i}\right) d x_{i}+\int_{P_{i}}^{\infty}\left(\theta_{i}-P_{i}\right) g\left(x_{i}\right) d x_{i}\right) \\
& =\sum_{i}\left(\int_{P_{i}}^{\infty}\left(x_{i}-\theta_{i}\right) \frac{1}{\sqrt{2 \pi} \sigma_{i}} e^{\frac{-\left(x_{i}-\theta_{i}\right)^{2}}{2 \sigma_{i}^{2}}} d x_{i}+\left(\theta_{i}-P_{i}\right)\left[1-\Phi\left(z_{i}\right)\right]\right) \\
& =\sum_{i}\left(\int_{z_{i}}^{\infty} y_{i} \frac{1}{\sqrt{2 \pi}} e^{\frac{-y_{i}^{2}}{2}} d y_{i} \sigma_{i}+\left(\theta_{i}-P_{i}\right)\left[1-\Phi\left(z_{i}\right)\right]\right) \quad y_{i}=\frac{x_{i}-\theta_{i}}{\sigma_{i}} \quad \& \quad d x_{i}=\sigma_{i} d y_{i} \\
& =\sum_{i}\left(\sigma_{i} \int_{z_{i}}^{\infty} \frac{1}{\sqrt{2 \pi}} e^{\frac{-y_{i}^{2}}{2}} d\left(\frac{y_{i}^{2}}{2}\right)+\left(\theta_{i}-P_{i}\right)\left[1-\Phi\left(z_{i}\right)\right]\right) \\
& =\sum_{i}\left(\left.\sigma_{i} \frac{1}{\sqrt{2 \pi}} e^{\frac{-y_{i}^{2}}{2}}\right|_{y_{i}=z_{i}}+\left(\theta_{i}-P_{i}\right)\left[1-\Phi\left(z_{i}\right)\right]\right) \\
& =\sum_{i}\left(\sigma_{i} \phi\left(z_{i}\right)+\left(\theta_{i}-P_{i}\right)\left[1-\Phi\left(z_{i}\right)\right]\right) \\
& =\sum_{i}\left(\left(\theta_{i}-P_{i}\right)\left[1-\Phi\left(z_{i}\right)\right]+\sigma_{i} \phi\left(z_{i}\right)\right)
\end{aligned}
$$

$$
\mathrm{i}=1 \ldots 12
$$

The terms in Eq. (A.6) can be calculated with an available commercial routine. 


\section{References}

[1] Jung J Y, Blau G, Pekny J F, Reklaitis G V, Eversdyk D. A simulation based optimisation approach to supply chain management under demand uncertainty. Computers \& Chemical Engineering 2004; 28 (10): 2087-2106.

[2] Gupta A, Maranas C D. A two-stage modeling and solution framework for multisite midterm planning under demand uncertainty. Ind. Eng. Chem. Res. 2000; 39: 3799.

[3] Henrion R, Li P, Möller A, Steinbach M, Wendt M, Wozny G. Stochastic optimization for chemical processes under uncertainty. Online Optimization of Large Scale Systems, Grötschel et al. eds., Springer-Verlag 2001; 455-476.

[4] Kall P, Wallace S W. Stochastic programming. New York: Wiley, 1994.

[5] Zimmermann H J. An application-oriented view of modeling uncertainty. Eur. J. Oper. Res. 2000; 122: 190-198.

[6] Prékopa A. Stochastic programming. Dordrecht: Kluwer, 1995.

[7] Uryasev S (Ed.). Probabilistic constrained optimization: methodology and applications. Dordrecht: Kluwer Academic Publishers, 2000.

[8] Li P, Wendt M, Wozny G. Optimal operations planning under uncertainty by using probabilistic programming. FOCAPO 2003. p. 289-292.

[9] Li P, Wendt M, Wozny G. Optimal production planning under uncertain market conditions. The $8^{\text {th }}$ PSE Kunming China, 2004. p. 511-516.

[10] Chan P, Cheung K Y, Hui C W, Sakamoto H, Hirata K. Electricity contract optimization for a large-scale chemical production site. The $8^{\text {th }}$ PSE Kunming China, 2004. p. 410-415.

[11] Brooke A, Kendrick D, Meeraus A. GAMSsA user's guide. The Scientific Press: Redwood City. CA, 1988.

[12] IBM. OSL (Optimization Subroutine Library) guide and reference (Release 2). Kingston. NY, 1991.

[13] Ochi M K. Applied probability and stochastic processes in engineering and physical sciences. New York: Wiley, 1990.

[14] Hopp W J, Spearman M L. Factory Physics $2^{\text {nd }}$ Edition. McGraw-Hill 2000: Chapter 2. p. 101-103. 


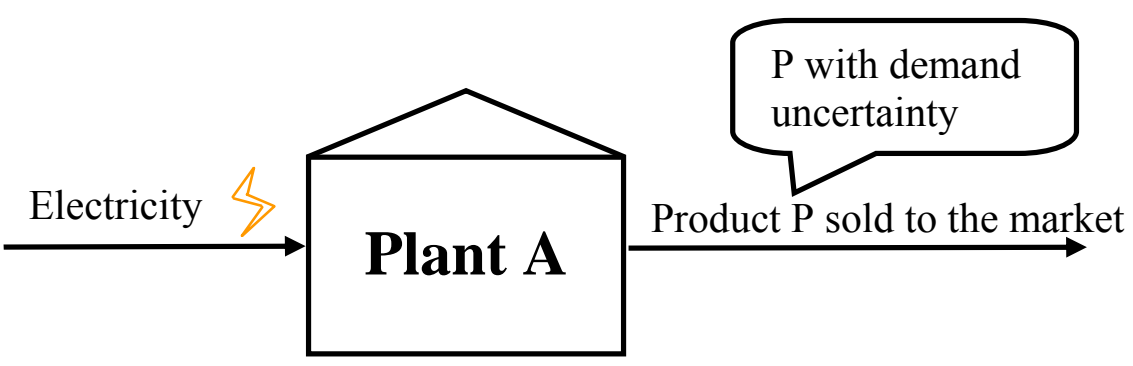

Fig. 1. Process network for the motivating example. 


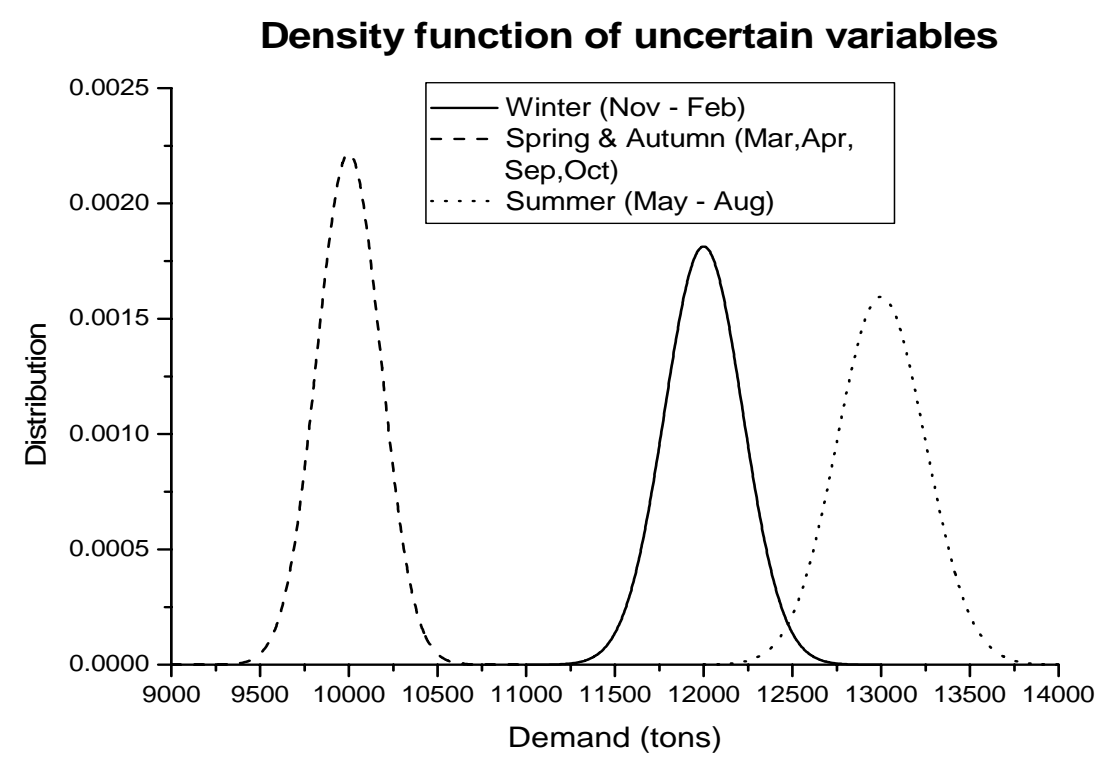

Fig. 2. Density function of the uncertain variable in the case study. 


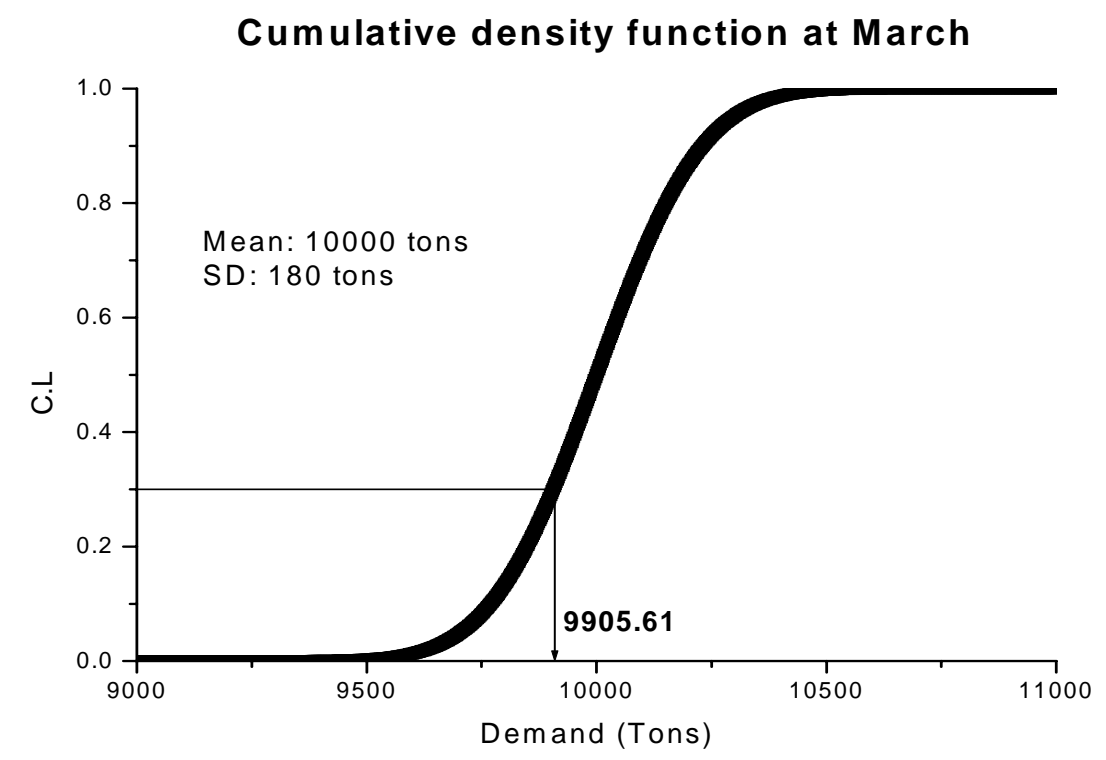

Fig. 3. The cumulative density function at March. 


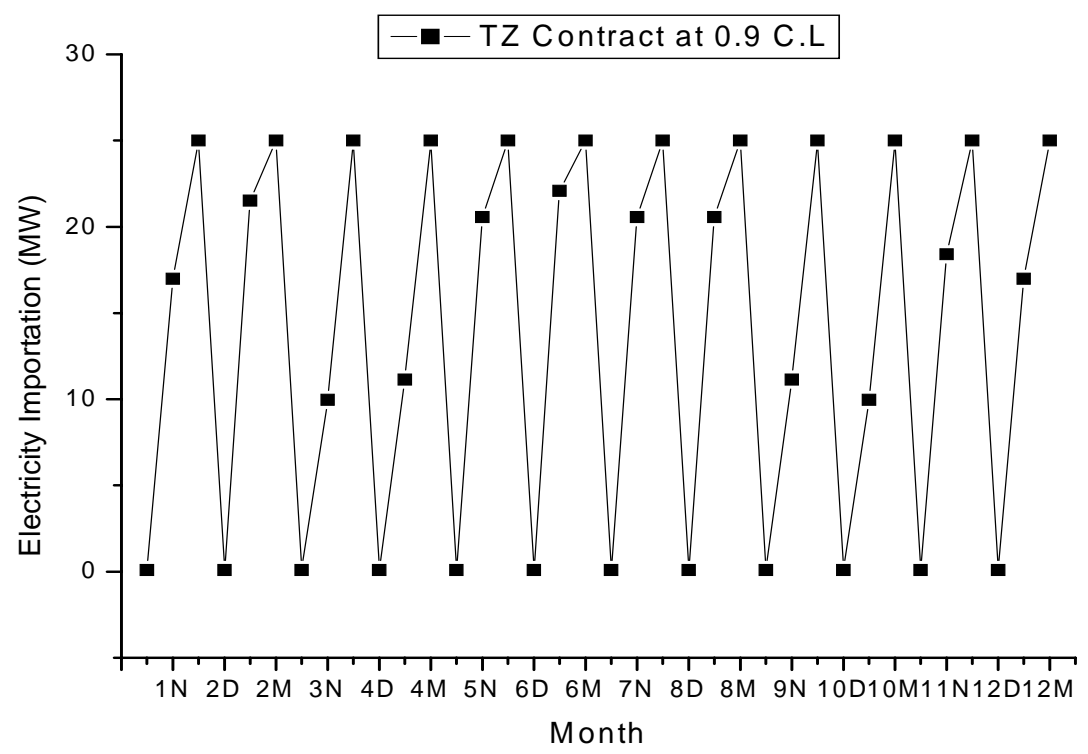

Fig. 4. Electricity importation Vs. Month of TZ contract. 


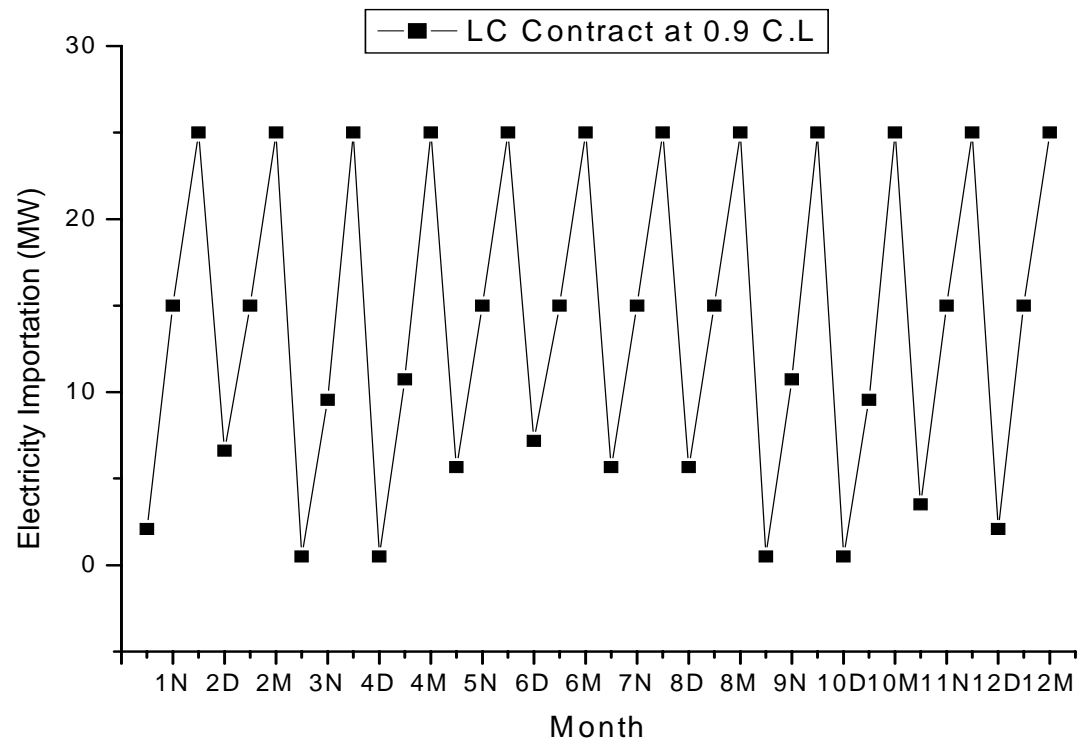

Fig. 5. Electricity importation Vs. Month of LC contract. 
The Profit Vs C.L

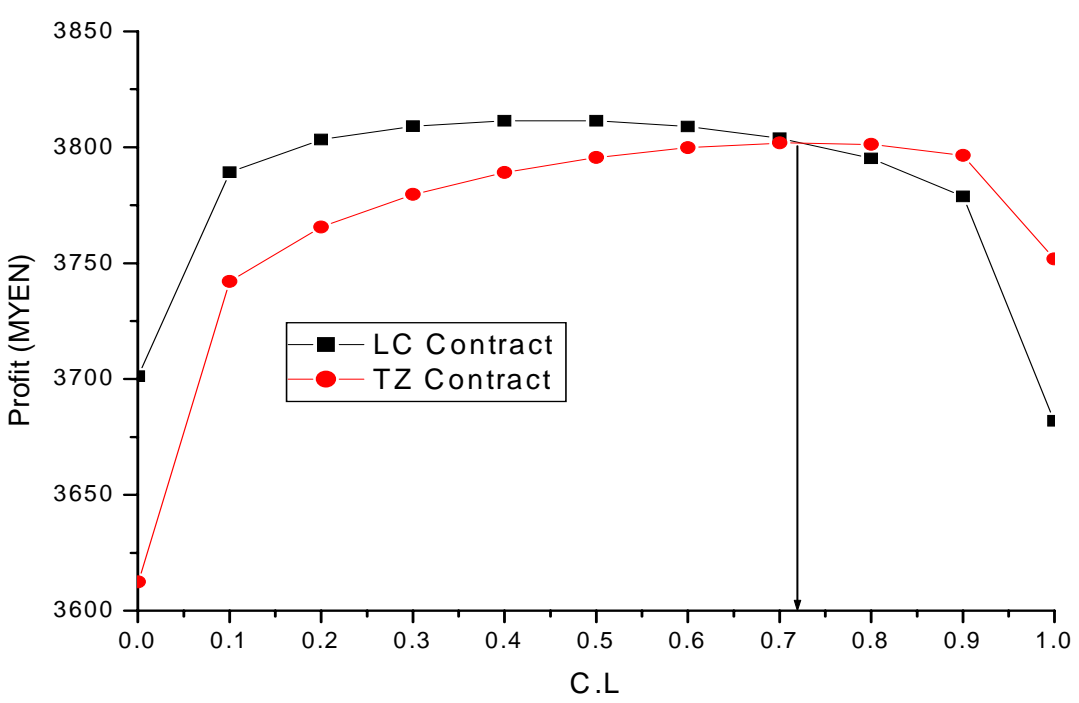

Fig. 6: The profit Vs. Confidence level. 
The Profit Vs C.L

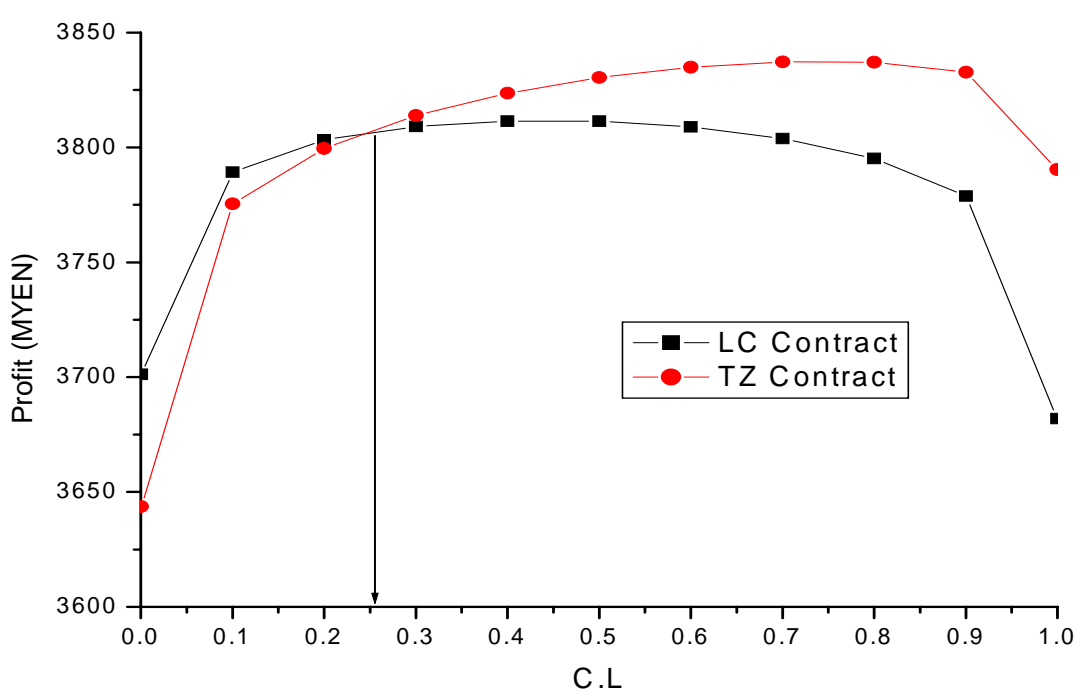

Fig. 7: Profit Vs. Confidence level with the second set of electricity price for the $\mathrm{TZ}$ contract. 
Table 1

The Mean of production target of product and its standard deviation

\begin{tabular}{lccc}
\hline Month & $\begin{array}{c}\text { Winter } \\
(\text { Nov }- \text { Feb })\end{array}$ & $\begin{array}{c}\text { Spring \& Autumn } \\
(\text { Mar, Apr, Sep, Oct })\end{array}$ & $\begin{array}{c}\text { Summer } \\
(\text { May }- \text { Aug) }\end{array}$ \\
\hline Mean $\theta(\mathrm{t})$ & 12000 & 10000 & 13000 \\
Standard Deviation $\sigma(\mathrm{t})$ & 220 & 180 & 250 \\
\hline
\end{tabular}


Table 2

Electricity price in LC and TZ contract

\begin{tabular}{ccc}
\hline Shift & \multicolumn{2}{c}{ Electricity Price, $\mathrm{C}_{\mathrm{ij}}(\mathrm{YEN} / \mathrm{MWh})$} \\
& LC contract & TZ contract \\
\hline $\mathrm{D}$ & 18000 & 21000 \\
$\mathrm{~N}$ & 6000 & 11000 \\
$\mathrm{M}$ & 3000 & 7700 \\
\hline
\end{tabular}


Table 3

Upper and lower bound of electricity importation in LC and TZ contract

\begin{tabular}{ccc}
\hline Shift & $\begin{array}{c}\text { Upper bound of electricity importation (MWh/shift) } \\
\text { LC contract }\end{array}$ & TZ contract \\
\hline D & 10 & 25 \\
N & 15 & 25 \\
M & 25 & \\
The lower bound of electricity importation in each shift is applied for the LC contract, \\
which is equal to $0.5 \mathrm{MW}$
\end{tabular}


Table 4

Fixed electricity cost calculation for the LC contract

$\mathrm{S}_{\mathrm{D}}, \mathrm{S}_{\mathrm{N}}, \mathrm{S}_{\mathrm{M}}$ Maximum of normal EL import at $\mathrm{D}, \mathrm{N}$ and $\mathrm{M}$ shifts (MW).

Fixed cost parameter of the normal EL import at $\mathrm{D}, \mathrm{N}$ and $\mathrm{M}$ shifts

$\mathrm{a}_{\mathrm{D}}, \mathrm{a}_{\mathrm{N}}, \mathrm{a}_{\mathrm{M}}$

(Day: 14, Night: 3, Mid-night: 1.5, MYEN/MW).

TEL Total electricity import (MWh), at least 100,000 MWh.

$\mathrm{a}_{\mathrm{T}}$

Cost parameter for total electricity import (0.0025 MYEN/MWh). 
Table 5

Data for Example 1

\begin{tabular}{ccc}
\hline Shift & Annual EL importation (MWh) & Max EL importation in each shift (MW) \\
\hline D & 500 & 0.3 \\
N & 1000 & 0.4 \\
M & 2000 & 0.7 \\
\hline
\end{tabular}


Table 6

Data for Example 2

\begin{tabular}{ccc}
\hline Shift & Annual EL importation (MWh) & Max EL importation in each shift (MW) \\
\hline D & 300 & 0.3 \\
N & 700 & 0.4 \\
M & 1500 & 0.7 \\
\hline
\end{tabular}


Table 7

The Production Target of product at different confidence levels

\begin{tabular}{cccc}
\hline Confidence level & $\begin{array}{c}\text { Winter } \\
\text { (Nov }- \text { Feb })\end{array}$ & $\begin{array}{c}\text { Spring \& Autumn } \\
(\text { Mar, Apr, Sep, Oct })\end{array}$ & $\begin{array}{c}\text { Summer } \\
(\text { May }- \text { Aug })\end{array}$ \\
\hline 0.0005 & 11276.07 & $(\mathrm{~T})$ & 12177.24 \\
0.10 & 11718.06 & 9407.63 & 12679.61 \\
0.20 & 11814.84 & 9769.32 & 12789.59 \\
0.30 & 11884.63 & 9848.51 & 12868.90 \\
0.40 & 11944.26 & 9905.61 & 12936.66 \\
0.50 & 12000.00 & 10000.00 & 13000.00 \\
0.60 & 12055.74 & 10045.60 & 13063.34 \\
0.70 & 12115.37 & 10094.39 & 13131.10 \\
0.80 & 12185.16 & 10151.49 & 13210.41 \\
0.90 & 12281.94 & 10230.68 & 13320.39 \\
0.9995 & 12724.02 & 10592.36 & 13822.70
\end{tabular}

"Notice that 0.0005 and 0.9995 are used as the minimum and maximum confidence levels instead of 0 and 1 . Hence $99.9 \%$ cases would be covered. 
Table 8

Expected Revenue with respect to confidence level

\begin{tabular}{cc}
\hline Confidence level & Expected Revenue (MYEN) \\
\hline 0.0005 & 4600.52 \\
0.1 & 4779.07 \\
0.2 & 4813.25 \\
0.3 & 4834.96 \\
0.4 & 4851.01 \\
0.5 & 4863.70 \\
0.6 & 4874.06 \\
0.7 & 4882.68 \\
0.8 & 4889.84 \\
0.9 & 4895.69 \\
0.9995 & 4899.99 \\
\hline
\end{tabular}


Table 9

Electricity cost with respect to confidence level under LC and TZ contracts and the total electricity import

\begin{tabular}{|c|c|c|c|}
\hline \multirow{2}{*}{ Confidence level } & \multicolumn{2}{|c|}{ Electricity cost (MYEN) } & \multirow{2}{*}{$\begin{array}{c}\text { EL imported } \\
\text { (MW) }\end{array}$} \\
\hline & LC contract & $\mathrm{TZ}$ contract & \\
\hline 0.0005 & 899.25 & 991.02 & 111727.19 \\
\hline 0.10 & 989.79 & 1039.87 & 116167.77 \\
\hline 0.20 & 1009.97 & 1050.56 & 117140.01 \\
\hline 0.30 & 1025.91 & 1058.27 & 117841.07 \\
\hline 0.40 & 1039.56 & 1064.86 & 118440.10 \\
\hline 0.50 & 1052.32 & 1071.02 & 119000.00 \\
\hline 0.60 & 1065.08 & 1077.18 & 119559.90 \\
\hline 0.70 & 1078.73 & 1083.77 & 120158.93 \\
\hline 0.80 & 1094.70 & 1091.48 & 120859.98 \\
\hline 0.90 & 1116.85 & 1102.17 & 121832.23 \\
\hline 0.9995 & 1218.04 & 1151.02 & 126272.86 \\
\hline
\end{tabular}


Table 10

Product rate

\begin{tabular}{cccc}
\hline$\alpha$ & Nov - Feb $(\mathrm{t})$ & Mar, Apr, Sep, Oct $(\mathrm{t})$ & May - Aug $(\mathrm{t})$ \\
\hline 0.9 & 12281.94 & 10230.68 & 13320.39 \\
\hline
\end{tabular}


Table 11

Amount of electricity to be purchased

\begin{tabular}{cccc}
\hline & TZ contract & \multicolumn{2}{c}{ LC contract } \\
\hline \multirow{2}{*}{ Shift } & Annual EL Imported & Annual EL Imported & Max EL Imported \\
& $(\mathrm{MW})$ & $(\mathrm{MW})$ & $(\mathrm{MW})$ \\
$\mathrm{D}$ & 292.00 & 9775.92 & 7.18 \\
$\mathrm{~N}$ & 48540.74 & 39056.31 & 15.0 \\
$\mathrm{M}$ & 73000.00 & 73000.00 & 25.0 \\
\hline
\end{tabular}


Table 12

The profit Vs. Confidence level

\begin{tabular}{ccc}
\hline \multirow{2}{*}{ Confidence level } & \multicolumn{2}{c}{ Profit (MYEN) } \\
\hline 0.0005 & LC contract & TZ contract \\
0.10 & 3701.27 & 3609.50 \\
0.20 & 3789.28 & 3739.20 \\
0.30 & 3803.28 & 3762.69 \\
0.40 & 3809.04 & 3776.69 \\
0.50 & $3811.45^{\#}$ & 3786.15 \\
0.60 & 3811.38 & 3792.68 \\
0.70 & 3808.99 & 3796.88 \\
0.80 & 3803.95 & $3798.91^{\#}$ \\
0.90 & 3795.14 & 3798.36 \\
0.9995 & 3778.84 & 3793.52 \\
\hline
\end{tabular}

\# The maximum profit obtained by the contract. 